\title{
Obesity, adipocyte hypertrophy, fasting glucose, and resistin are potential contributors to nonalcoholic fatty liver disease in South Asian
} women

This article was published in the following Dove Press journal:

Diabetes, Metabolic Syndrome and Obesity: Targets and Therapy

\author{
Kembra Albracht-Schulte' \\ Shanthini Rosairo ${ }^{2}$ \\ Latha Ramalingam' \\ Sulochana Wijetunge ${ }^{3}$ \\ RMCJ Ratnayake ${ }^{4}$ \\ HMSRB Kotakadeniya ${ }^{5}$ \\ John A Dawson' \\ Nishan S Kalupahana ${ }^{1,6}$ \\ Naima Moustaid-Moussa' \\ 'Department of Nutritional Sciences and \\ Obesity Research Institute, Texas Tech \\ University, Lubbock, TX, USA; \\ ${ }^{2}$ Department of Radiology, University of \\ Peradeniya, Peradeniya, Sri Lanka; \\ ${ }^{3}$ Department of Pathology, University of \\ Peradeniya, Peradeniya, Sri Lanka; \\ ${ }^{4}$ Department of Obstetrics and \\ Gynecology, University of Peradeniya, \\ Peradeniya, Sri Lanka; ${ }^{5}$ Department of \\ Surgery, University of Peradeniya, \\ Peradeniya, Sri Lanka; ' ${ }^{6}$ Department of \\ Physiology, Faculty of Medicine, \\ University of Peradeniya, Peradeniya, Sri \\ Lanka
}

Purpose: Nonalcoholic fatty liver disease (NAFLD) is often referred to as the hepatic manifestation of the metabolic syndrome. The relationship between body weight, NAFLD, and insulin resistance is not well characterized in humans. Additionally, it is unclear why South Asians develop these complications at lower levels of obesity compared to their Western counterparts.

Patients and methods: To address this question, we performed a cross-sectional study using a convenience sample of Sri Lankan adult females $(n=34)$ and collected anthropometric data, adipose tissue specimens (for histology), and fasted serum samples (for metabolic and inflammatory markers). Hepatic steatosis was assessed by ultrasound scanning and used to classify participants as NAFL 0, NAFL 1, and NAFL 2.

Results: Waist circumference significantly increased with increasing NAFL grade. Participants with NAFL had significantly higher body mass index, hip circumference, and fasting plasma glucose, as well as a higher mean adipocyte area in both abdominal subcutaneous and visceral areas, indicating a higher degree of adipocyte hypertrophy associated with fatty liver. There were, however, no differences in measures of dyslipidemia. Of the multiple adipokines measured, resistin was the only proinflammatory adipokine significantly elevated in NAFL 2.

Conclusion: These findings indicate that measures of adiposity, fasting serum glucose, and resistin may be important indicators of NAFLD in South Asian women.

Keywords: adipokines, inflammation, metabolic syndrome

\section{Introduction}

Nonalcoholic fatty liver disease (NAFLD) is the most common cause of chronic liver disease worldwide and is increasing in prevalence due to increasing obesity rates in both children and adults. ${ }^{1-4}$ NAFLD represents a range of hepatic pathology in the absence of excessive alcohol consumption and/or other causes of chronic liver disease. Simple steatosis, or NAFL, characterized by the presence of $>5 \%$ triglycerides (TGs) in hepatocytes progresses to nonalcoholic steatohepatitis (NASH) due to inflammation and collagen deposition, ${ }^{5}$ which is strongly linked to liver-related morbidity and mortality. ${ }^{6}$

NAFLD is often referred to as the hepatic manifestation of the metabolic syndrome, which is characterized by at least three of the following: hypertension,
Correspondence: Naima Moustaid-Moussa Department of Nutritional Sciences, Obesity Research Institute, College of Human Sciences, Texas Tech University, I30I Akron Street, Lubbock, TX 79409-1270, USA Tel +l 8068347946

Email naima.moustaid-moussa@ttu.edu 
hyperglycemia, abdominal obesity, elevated plasma TGs, and reduced plasma high-density lipoproteins (HDL). ${ }^{7}$ Although NAFLD pathogenesis is not well established, it is clear that obesity and insulin resistance are key contributors. ${ }^{8}$ Obesity increases the release of free fatty acids (FFAs) from adipose tissue into hepatocytes, contributing to more than two-thirds of FFAs in the liver, ${ }^{9}$ which aids in systemic insulin resistance. ${ }^{10}$ Furthermore, excessive adipose tissue increases proinflammatory adipokine secretion (eg, leptin, resistin, IL-6) that may 1) directly impact the liver and contribute to NAFLD pathogenesis ${ }^{11}$ and 2) cause systemic inflammation, which contributes to insulin resistance. ${ }^{12}$ Insulin resistance exacerbates metabolic abnormalities, including dyslipidemia and obesity, ${ }^{13}$ and thus induces NAFLD development. ${ }^{14}$

Interestingly, not all individuals with metabolic syndrome will develop NAFLD and not all cases of fatty liver will progress to NASH or end-stage liver disease. ${ }^{5}$ Susceptibility to steatosis, and its associated metabolic consequences, as well as NAFLD progression have all been linked with ethnic and sex differences. ${ }^{15}$ Further, the association of NAFLD and obesity also varies by ethnicity, with Asian individuals at a higher risk of what is considered "nonobese NAFLD", but that may be more strongly linked to central adiposity, genetic predisposition and diet. ${ }^{16,17}$

Accordingly, $9.2 \%$ of Sri Lankan adults were obese, while $26.2 \%$ were considered centrally obese in $2010 .{ }^{18}$ A metaanalytic analyses of epidemiological data suggests NAFLD prevalence in Asia to be $27 \% .{ }^{19}$ Compared to Caucasians, South Asians have a higher amount of total body fat, including subcutaneous adipose tissue and especially visceral adipose tissue for a given body mass index (BMI). ${ }^{20,21}$ At a comparatively low BMI, South Asians have an increased risk of hyperlipidemia, hypertension, glucose intolerance, and NAFLD, in part, due to excessive central adiposity ${ }^{22-24}$ but also due to genetic predisposition and excess energy consumption. ${ }^{17}$

Since the presence of NALFD is linked to abdominal obesity and South Asians are at an increased risk of developing obesity-related metabolic abnormalities, it is of interest to dissect alterations in the metabolic profile that exist with varying degrees of NAFLD in this population. Furthermore, no single biomarker or group of metabolites has been related to the progression of NAFLD in human serum, and thus proper medical care for NAFLD is limited. ${ }^{25}$

Therefore, the purpose of the current study was to determine the association between degree of adiposity, plasma adipokine levels, and markers of inflammation and insulin resistance with the presence of fatty liver in a South Asian population. We hypothesized that an increase in insulin resistance, markers of inflammation, and serum adipokines would exist proportional to the degree of fatty liver.

\section{Material and methods}

This was a cross-sectional study to determine the association between anthropometric measures, measures of adiposity, indices of systemic insulin resistance, and plasma adipokine levels with degree of hepatic steatosis in Sri Lankan adults. Ethical clearance for this study was granted by the Faculty of Medicine, University of Peradeniya and informed written consent was obtained from all participants. De-identified serum samples were processed at Texas Tech University under an exempt Institutional Review Board (IRB) Approval (IRB \#503875).

\section{Participants}

A convenience sample of adult women (age 18 to 65 years) undergoing routine abdominal surgery (hernia repair $n=4$; hysterectomy $n=10$; laparoscopy $n=6$; laparotomy $\mathrm{n}=3$; other $\mathrm{n}=11$ ) at the Teaching Hospital, Peradeniya, Sri Lanka, were recruited $(\mathrm{n}=34)$. Individuals with inflammatory conditions, cancer, or previous abdominal surgery were excluded from the study. Demographic data, diet, and physical activity history were recorded using an interviewer-administered questionnaire.

On the day of the surgery, a fasting blood sample was collected, and a radiologist performed an ultrasound scan of the abdomen. Blood was collected into microcentrifuge tubes and allowed to clot on ice. Serum was separated via centrifugation and supernatant was stored in $-80^{\circ} \mathrm{C}$ for subsequent analyses.

\section{Anthropometric measurements}

Height was measured to the nearest millimeter using a stadiometer. Weight was measured to the nearest 100 $\mathrm{g}$ with a digital scale. Waist circumference (WC) was measured midway between the lowest rib and the superior border of the iliac crest in the mid-axillary line, with an inelastic measuring tape at the end of normal expiration to the nearest millimeter. Hip circumference (HC) was measured around the widest portion of the buttocks. BMI was calculated with the weight $(\mathrm{wt})$ and height $(\mathrm{ht})$ data $\left[\mathrm{BMI}=\mathrm{wt}(\mathrm{kg}) / \mathrm{ht}^{2}\left(\mathrm{~m}^{2}\right)\right]$.

\section{Liver ultrasonography}

A radiologist performed an ultrasound scan of the liver. A $3.5-\mathrm{MHz}$ transducer was used to obtain the following 
images: sagittal view of the right lobe of the liver and right kidney, transverse view of the left lateral segment of the liver and spleen, transverse view of the liver and pancreas, and any focal areas of altered echotexture (Philips EnVisor C HD M2540A-USA-Diagnostic Ultrasound Imaging System, Andover, MA, USA). The severity of echogenicity was graded as follows: fatty liver grade 0 (NAFL 0 , normal echogenicity); fatty liver grade 1 (NAFL 1, slight, diffuse increase in fine echoes in liver parenchyma with normal visualization of diaphragm and intrahepatic vessel borders); fatty liver grade 2 (NAFL 2, moderate, diffuse increase in fine echoes with slightly impaired visualization of intrahepatic vessels and diaphragm); and fatty liver grade 3 (NAFL 3, marked increase in fine echoes with poor or nonvisualization of the intrahepatic vessel borders, diaphragm, and posterior right lobe of the liver). ${ }^{26}$

\section{Adipose tissue histology}

Adipose tissue samples were obtained from the anterior abdominal wall close to the umbilicus (subcutaneous adipose tissue) and from the greater omentum (visceral adipose tissue). Samples were formalin fixed, and a routine paraffin embedded tissue section was prepared. Adipocyte size was measured using the ImageJ (NIH, http://imagej.nih.gov/ij/) software (mean surface area of 100 adipocytes in each biopsy).

\section{Serum biomarkers and adipokines Enzymes}

Enzymes indicative of liver damage, including alanine aminotransferase (ALT) and aspartate aminotransferase (AST) enzymes, were assayed using IFCC method in an automated biochemical analyzer (Thermo Scientific Konelab Analyzer Holliston, MA, USA).

\section{Measures of insulin resistance}

Blood glucose was measured by the glucose oxidase method. HOMA index was calculated as fasting glucose $(\mathrm{mmol} / \mathrm{L}) \dot{x}$ fasting insulin $(\mathrm{mU} / \mathrm{L}) / 22.5$.

\section{Branched-chain amino acids}

Colorimetric Assay Kit (BioVision Incorporated, Milpitas, CA, USA) was used to measure branched-chain amino acids (BCAAs), leucine, isoleucine, and valine (Cat. \#K564-100; range $=0-10 \mathrm{nmol}){ }^{27}$

\section{Lipids}

TGs, HDL, very-low-density lipoprotein (VLDL), and lowdensity lipoprotein (LDL) were assessed by CovenantHealth (Lubbock, TX, USA).

\section{Hormones and adipokines}

Luminex XMAP technology Magpix ${ }^{\mathrm{TM}}$, Human Adipokine Magnetic Bead Panel I (Millipore, Billerica, MA, USA; Cat. \# HADK1MAG-61K) was used to measure adiponectin (range $=26-400,000 \mathrm{ng} / \mathrm{mL}$ ) and resistin (range $=6.4-100,000 \mathrm{pg} / \mathrm{mL}) .^{28}$ Milliplex $^{\circledR}$ Human Adipokine Magnetic Bead Panel II (EMD Millipore, Billerica, MA, USA; Cat. \# HADK2MAG-61K) was used to measure IL-6 (range $=0.96-15,000 \mathrm{pg} / \mathrm{mL}$ ), leptin (range $=38-600,000 \mathrm{pg} / \mathrm{mL}$ ), and monocyte chemoattractant protein-1 (MCP-1; range $=1.3-20,000 \mathrm{pg} / \mathrm{ml}) .{ }^{29}$ BioTek Cytation imaging reader technology, ELISA (EMD Millipore) was used to measure high-molecularweight (HMW) adiponectin (Cat. \#SPREZHMWADPN65K; range $=1.56-200.88 \mathrm{ng} / \mathrm{mL}$ ) and insulin (Cat. \#EZHI-14K; range $=2-200 \mu \mathrm{U} / \mathrm{mL}$ ). ${ }^{30}$ Angiotensinogen (AGT) (Cat. \#ELH-AGT; range $=1.229-300 \mathrm{ng} / \mathrm{mL}$ ) was measured using a kit from RayBiotech (Norcross, GA, USA). High-sensitivity C-reactive protein (hsCRP) was measured using a kit from MP Biomedicals (Solon, OH, USA; Cat. \# 07BC1119 ; range $=0-0.1 \mathrm{mg} / \mathrm{L}$ ). When appropriate, serum dilutions were performed.

\section{Statistical analysis}

Data were entered using Microsoft Excel (2010) and an ANOVA was performed using $\mathrm{R}$ data analysis software, version 20 (Statistical and Products Service Solutions, Chicago, IL, USA). Bonferroni-Holm corrections were performed. When necessary, post-hoc comparisons were made. Differences were considered significant at $p<0.05$ after the aforementioned correction.

\section{Results}

\section{Participants}

Of the 34 female participants, 11 were categorized as fatty liver grade 0 (NAFL 0), 13 were categorized as fatty liver grade 1 (NAFL 1), 10 were categorized as fatty liver grade 2 (NAFL 2), and 0 were categorized as fatty liver grade 3 (NAFL 3). Participant characteristics are presented in Table 1. Age of the participants was not significantly different based on the degree of steatosis. The number of participants with hypertension did not vary among fatty liver groups.

\section{NAFL and measures of adiposity}

After Bonferroni-Holm corrections, WC $(p=0.00027)$ was the only significant variable increasing across NAFL grade: 
Table I Characteristics of subjects with varying degrees of fatty liver

\begin{tabular}{|c|c|c|c|c|}
\hline & $\begin{array}{l}\text { NAFL } \\
\text { Grade } 0 \\
(n=I I)\end{array}$ & $\begin{array}{l}\text { NAFL } \\
\text { Grade I } \\
(n=13)\end{array}$ & $\begin{array}{l}\text { NAFL } \\
\text { Grade } 2 \\
(n=10)\end{array}$ & $p$-value \\
\hline $\begin{array}{l}\text { Age (years) } \\
\text { Body weight }(\mathrm{kg}) \\
\text { Height }(\mathrm{m}) \\
\text { BMI }\left(\mathrm{kg} / \mathrm{m}^{2}\right)\end{array}$ & $\begin{array}{l}45.00 \pm 3.18 \\
50.20 \pm 3.58 \\
1.53 \pm 0.02 \\
21.45 \pm 1.43\end{array}$ & $\begin{array}{l}46.15 \pm 4.80 \\
59.6 \pm 1.37 \\
1.51 \pm 0.02 \\
26.62 \pm 0.57\end{array}$ & $\begin{array}{l}46.70 \pm 4.25 \\
73.61 \pm 7.76 \\
1.55 \pm 0.01 \\
30.64 \pm 3.01\end{array}$ & $\begin{array}{l}p=0.96 \\
p=0.0043\end{array}$ \\
\hline $\begin{array}{l}\text { Markers of insulin resistance } \\
\text { Insulin }(\mu \mathrm{U} / \mathrm{mL}) \\
\text { HOMA-IR } \\
\text { BCAA (nmol) }\end{array}$ & $\begin{array}{l}3.99 \pm 1.24 \\
832.35 \pm 260.24 \\
6.94 \pm 0.50\end{array}$ & $\begin{array}{l}8.21 \pm 2.17 \\
1,927.24 \pm 499.79 \\
6.44 \pm 0.33\end{array}$ & $\begin{array}{l}5.91 \pm 1.36 \\
1,786.77 \pm 495.39 \\
7.77 \pm 0.41\end{array}$ & $\begin{array}{l}p=0.23 \\
p=0.23 \\
p=0.75\end{array}$ \\
\hline $\begin{array}{l}\text { Enzymes } \\
\text { ALT(U/L) } \\
\text { AST (U/L) } \\
\text { AST/ALT }\end{array}$ & $\begin{array}{l}26.52 \pm 10.68 \\
24.57 \pm 3.69 \\
1.38 \pm 0.22\end{array}$ & $\begin{array}{l}25.97 \pm 4.53 \\
28.77 \pm 3.93 \\
1.23 \pm 0.13\end{array}$ & $\begin{array}{l}39.22 \pm 10.81 \\
40.68 \pm 6.19 \\
1.27 \pm 0.14\end{array}$ & $\begin{array}{l}p=0.49 \\
p=0.066\end{array}$ \\
\hline $\begin{array}{l}\text { Lipids } \\
\text { Triglycerides (mg/dL) } \\
\text { HDL cholesterol (mg/dL) } \\
\text { VLDL cholesterol (mg/dL) } \\
\text { LDL cholesterol (mg/dL) } \\
\text { Hormones and adipokines } \\
\text { Adiponectin (total-ng/mL) } \\
\text { HMW adiponectin }(\mathrm{ng} / \mathrm{mL}) \\
\text { Leptin }(\mathrm{pg} / \mathrm{mL}) \\
\text { AGT }(\mathrm{ng} / \mathrm{ml})\end{array}$ & $\begin{array}{l}116 \pm 14.57 \\
23.11 \pm 3.72 \\
23.33 \pm 2.85 \\
100.89 \pm 6.96 \\
67,055.32 \pm 52,370.42 \\
9,650.16 \pm 3,341.15 \\
96,424.26 \pm 38,381.47 \\
126,368.6 \pm 26,347.95\end{array}$ & $\begin{array}{l}|4| .80 \pm|3.6| \\
|9.10 \pm 2.4| \\
28.40 \pm 2.64 \\
105.30 \pm 6.28 \\
|1,439.6| \pm 2,292.8 \mid \\
6,548.20 \pm \mid, 922.80 \\
60,009.89 \pm 2 \mid, 855.84 \\
101,292.46 \pm 23,88 \mid .53\end{array}$ & $\begin{array}{l}118.88 \pm \mid 1.45 \\
21.50 \pm 1.58 \\
23.75 \pm 2.32 \\
109.25 \pm 10.24 \\
8,418.93 \pm 805.26 \\
8,951.96 \pm 2,140.96 \\
59,258.26 \pm 29,391.08 \\
\mid 21,345 \pm 33,931.19\end{array}$ & $\begin{array}{l}p=0.33 \\
p=0.58 \\
p=0.32 \\
p=0.76 \\
p=0.27 \\
p=0.63 \\
p=0.62 \\
p=0.79\end{array}$ \\
\hline $\begin{array}{l}\text { Markers of inflammation } \\
\mathrm{MCP}-\mathrm{I}(\mathrm{pg} / \mathrm{mL}) \\
\mathrm{IL}-6(\mathrm{Pg} / \mathrm{mL}) \\
\text { hsCRP }(\mathrm{mg} / \mathrm{L})\end{array}$ & $\begin{array}{l}|4.9| \pm 7.2 \mid \\
9.07 \pm 4.25 \\
1.05 \pm 0.22\end{array}$ & $\begin{array}{l}8.93 \pm 1.93 \\
8.67 \pm 4.80 \\
4.57 \pm 1.91\end{array}$ & $\begin{array}{l}18.14 \pm 6.12 \\
10.12 \pm 3.82 \\
6.21 \pm 2.77\end{array}$ & $\begin{array}{l}p=0.44 \\
p=0.96 \\
p=0.15\end{array}$ \\
\hline
\end{tabular}

Note: Data presented as mean \pm SEM.

Abbreviations: AGT, angiotensinogen; ALT, alanine aminotransferase; AST, aspartate aminotransferase; BMI, body mass index; HMW, high molecular weight; HDL, highdensity lipoprotein; hsCRP, high-sensitivity C-reactive protein; LDL, low-density lipoprotein; MCP-I, monocyte chemoattractant protein I; NAFL, nonalcoholic fatty liver; VLDL, very-low-density lipoprotein.

post-hoc analyses indicate an increase between NAFL 0 and 1 $(p=0.006)$, NAFL 1 and NAFL $2(p=0.027)$, and also between NAFL 0 and $2(p=0.001)$ (Figure 1A). BMI $(p=0.0043)$ and $\mathrm{HC}(p=0.013)$ were nominally significant based on the degree of NAFL. Post-hoc analyses for BMI indicate a significant increase between NAFL 0 and NAFL $1(p=0.0014)$ and NAFL 0 and NAFL $2(p=0.011)$, but not between NAFL 1 and NAFL $2(p=0.13)$ (Figure 1B). Similarly, post-hoc analyses for HC indicate a significant increase between NAFL 0 and NAFL 1 $(p=0.002)$ and NAFL 0 and NAFL $2(p=0.022)$, but not between NAFL 1 and NAFL $2(p=0.27)$ (Figure 1C). There was not a significant difference in waist-to-hip ratio among the groups $(p=0.17)$ (Figure 1D). These findings indicate that increasing adiposity, especially in the visceral compartment, is associated with NAFLD.

\section{NAFL and adipose tissue dysfunction}

Adipose tissue dysfunction, indicated by dysregulated adipokine secretion and chronic low-grade inflammation, is mechanistically linked to the pathogenesis of obesity-associated metabolic disorders. Dysfunctional adipose tissue is characterized by large, insulin-resistant adipocytes. ${ }^{12}$ Thus, next, we measured adipocyte size in visceral and subcutaneous compartments to identify a link with NAFL. Abdominal subcutaneous adipocyte area was nominally significant among different NAFL groups $(p=0.0034)$, with post-hoc analyses indicating a significant increase between NAFL 0 and NAFL $2(p=0.005)$ and trending increases between NAFL 0 and NAFL $1(p=0.052)$ and NAFL 1 and NAFL $2(p=0.054)$ (Figure 2A). Representative images of subcutaneous abdominal adipocyte area are shown in Figure 2B. Visceral (omental) 


\section{A}

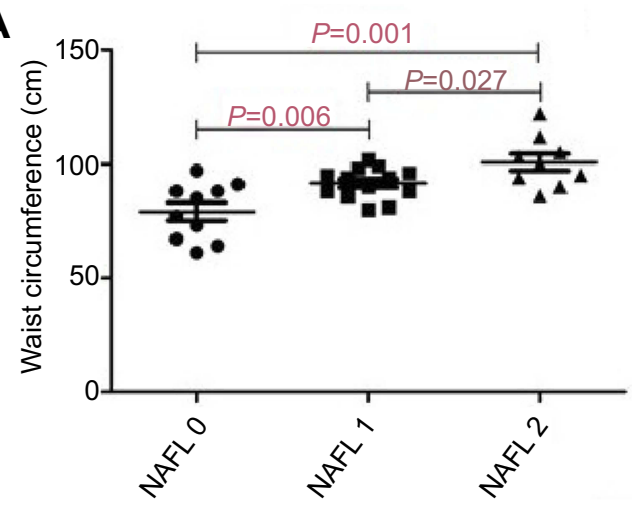

C

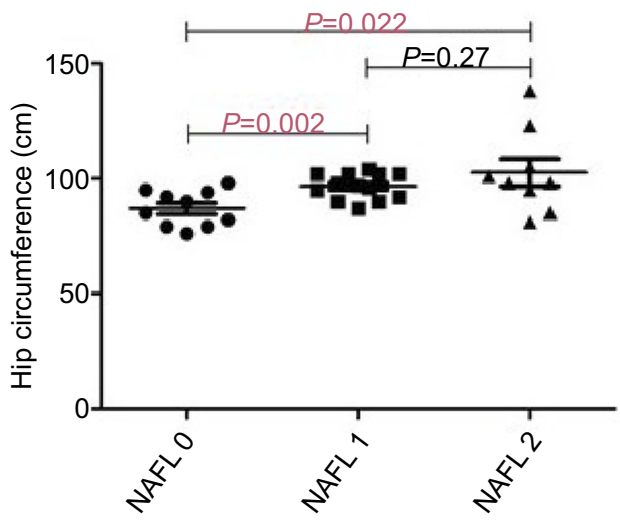

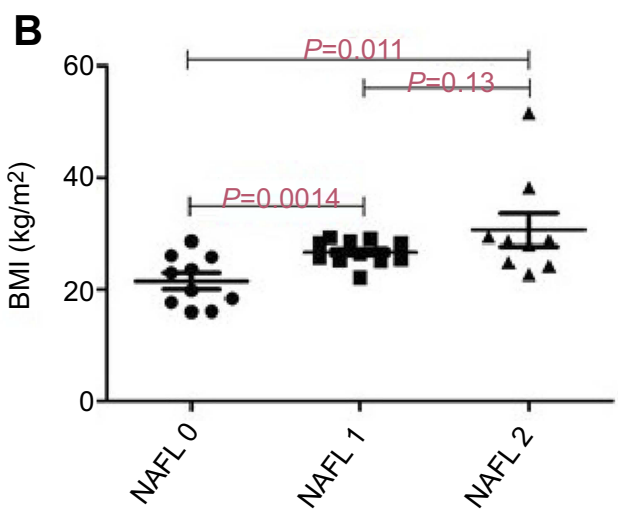

D

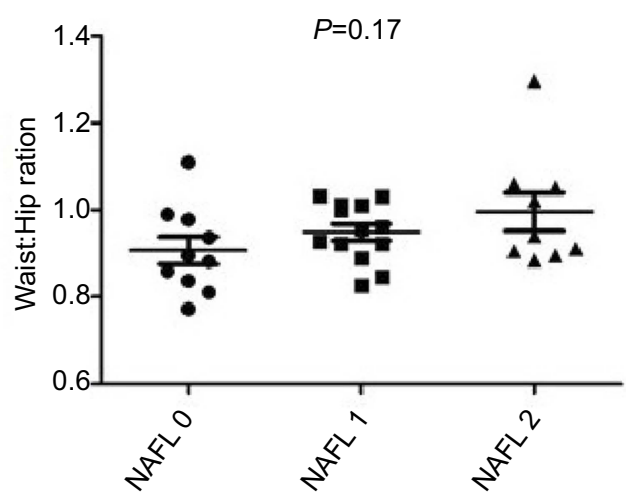

Figure I Anthropometric measures in participants with varying degree of nonalcoholic fatty liver (NAFL). (A) Participants with higher grade of fatty liver had a significantly higher waist circumference (WC). Post-hoc analyses indicate a significant increase in WC between each grade of NAFL. (B) Participants with fatty liver had a significantly higher body mass index (BMI) as shown by post-hoc analyses, which indicate a significant increase in BMI between NAFL 0 and NAFL I, but not between NAFL I and NAFL 2. (C) Participants with fatty liver had a significantly higher hip circumference as shown by post-hoc analyses, which indicate a significant increase in hip circumference between NAFL 0 and NAFL I, but not between NAFL I and NAFL 2. (D) Waist-to-hip ratio was not significantly different among fatty liver grade. Hepatic steatosis was assessed using ultrasound. $n=10-13$ per group. ANOVA with Bonferroni-Holm corrections analyses was performed.

adipocyte area was also nominally significant among different NAFL groups $(p=0.0042)$, with post-hoc analyses indicating a significant increase between NAFL 0 and NAFL $1(p=0.022)$ and NAFL 0 and NAFL $2(p=0.006)$, but not between NAFL 1 and NAFL $2(p=0.22)$ (Figure 2C).

\section{Serum biomarkers and adipokines NAFL and liver enzymes}

NAFL can lead to elevated liver enzymes. ${ }^{31,32}$ ALT was not statistically different among participants with varying degrees of NAFLD. There was a trend toward nominal significance for AST ( $p=0.066$ ) among NAFL groups, with post-hoc analyses indicating a significant increase in AST between NAFL 0 and NAFL $2(p=0.035)$, but not between NAFL 0 and NAFL 1 $(p=0.46)$ or NAFL 1 and NAFL $2(p=0.10)$ (Table 1$)$.

\section{NAFL and insulin resistance}

NAFLD is associated with insulin resistance. However, it is not clear whether the latter is a cause or effect of NAFLD. ${ }^{14}$ We found that fasting blood glucose $(\mathrm{FBG})(p=0.0052)$ nominally increased among NAFL groups (Figure 3). Posthoc analyses for FBG indicated a significant increase between NAFL 0 and NAFL $1(p=0.011)$ and NAFL 0 and NAFL $2(p=0.003)$, but not between NAFL 1 and NAFL 2 $(p=0.26)$ (Figure 3). Accordingly, participants in the NAFL 1 and NAFL 2 groups met criteria for prediabetes (5.6 to 6.9 $\mathrm{mmol} / \mathrm{L}){ }^{33}$ However, neither serum insulin nor HOMAIR were significantly different among participants with varying degrees of NAFL (Table 1). Since BCAAs are altered with insulin resistance, ${ }^{34}$ they were also assessed in our study but were not significantly different based on the degree of NAFL (Table 1). Taken together, these findings suggest that insulin resistance is unlikely to be the major mechanism responsible for NAFL in this population.

\section{NAFL and lipids}

All lipid panel measures, including VLDL, LDL, HDL, and serum TGs, were not significantly different based on the degree of NAFLD (Table 1). 
A

B
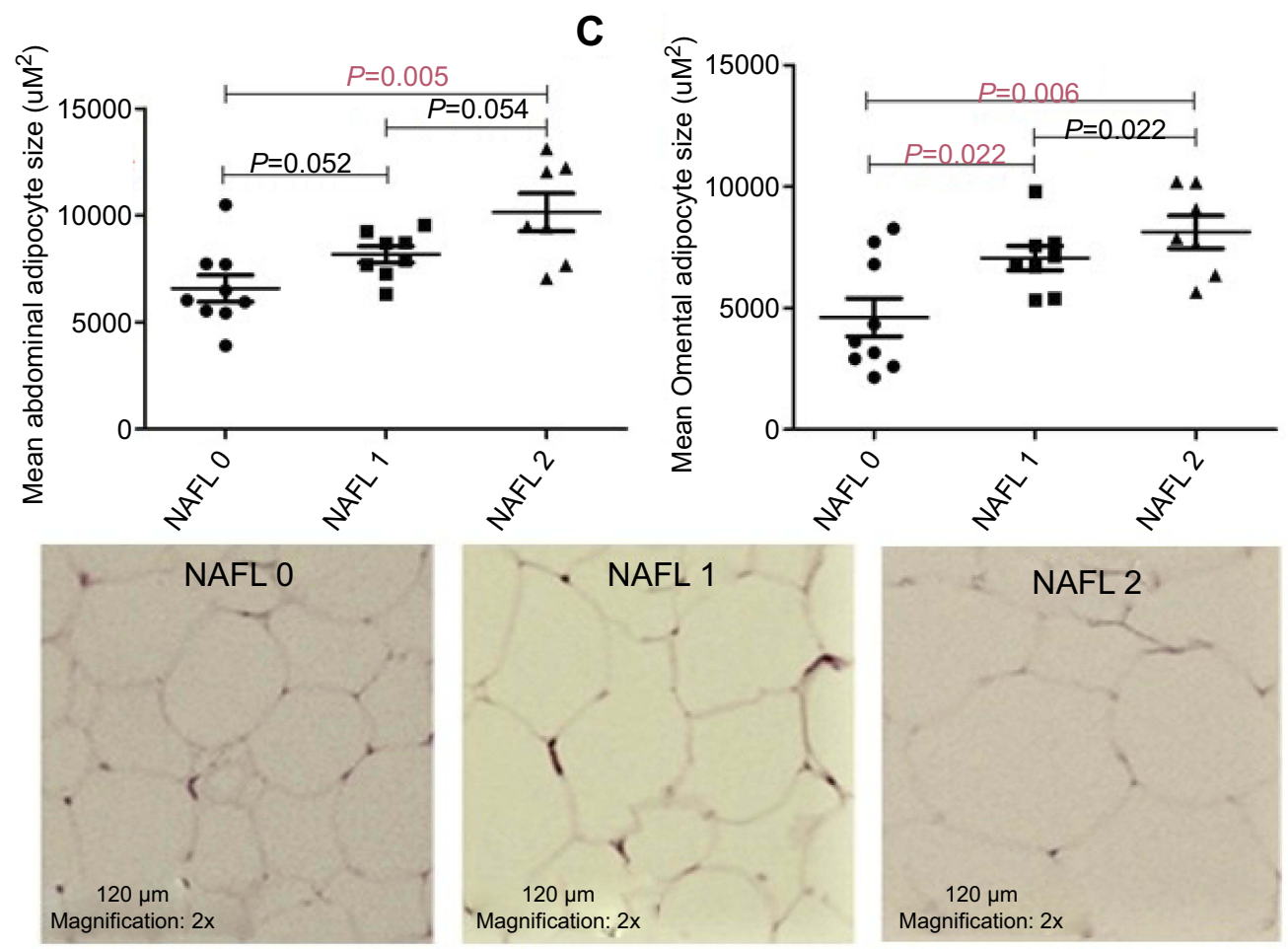

Figure 2 Mean adipocyte size in participants with varying degree of nonalcoholic fatty liver (NAFL). (A) Participants with NAFL 2 had a significantly higher abdominal adipocyte area as shown by post-hoc analyses, which also indicate increases in abdominal adipocyte area between NAFL 0 and NAFL I and NAFL I and NAFL 2 that are trending significance. (B) Representative H\&E-stained sections of abdominal adipose tissue (subcutaneous) are shown for each group. (C) Participants with fatty liver had a significantly higher omental (visceral) adipocyte area as shown by post-hoc analyses, which indicate a significant increase in adipocyte area between NAFL 0 and NAFL I, but not between NAFL I and NAFL 2. Hepatic steatosis was assessed using ultrasound. Adipocyte area was measured using the Image software. $n=10-13$ per group. ANOVA with Bonferroni-Holm corrections analyses was performed.

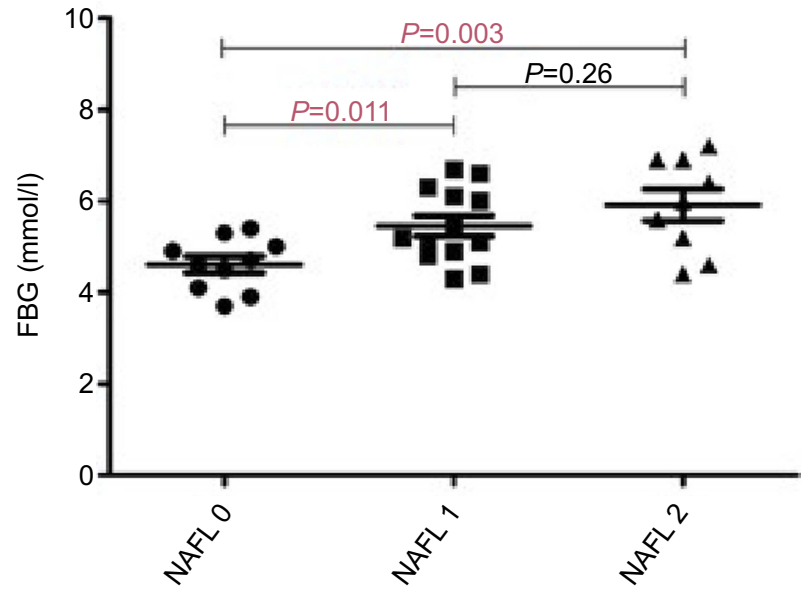

Figure 3 Elevated fasting blood glucose with degree of nonalcoholic fatty liver (NAFL). Participants with fatty liver had significantly higher fasting blood glucose (FBG) as shown by post-hoc analyses, which indicate a significant increase in FBG between NAFL 0 and NAFL I, but not between NAFL I and NAFL 2. Hepatic steatosis was assessed using ultrasound. $n=10-13$ per group. ANOVA with Bonferroni-Holm corrections analyses was performed.

\section{NAFL, hormones, and adipokines}

Obesity is characterized by a chronic low-grade inflammation. ${ }^{12}$ However, all markers of inflammation evaluated in our study, including hsCRP, MCP-1, and IL-6, were not significantly different based on the degree of NAFLD (Table 1), indicating that systemic inflammation is unlikely to be associated with NAFLD in this population.

Furthermore, adipokines, including adiponectin, HMW adiponectin, and leptin, were not significantly different based on NAFL degree (Table 1). There was a trend toward nominal significance for resistin $(p=0.063)$ among NAFL groups, with post-hoc analyses indicating a significant increase in serum resistin between NAFL 0 and NAFL $2(p=0.025)$ and a trend toward significance between NAFL 1 and NAFL $2(p=0.052)$, but not between NAFL 0 and NAFL $1(p=0.81)$ (Figure 4).

Activation of the renin-angiotensin system favors NAFLD development and progression. ${ }^{35}$ However, AGT was not significantly different based on the degree of NAFL in our sample population (Table 1).

\section{Discussion}

In this study, we found that in female Sri Lankan participants, fatty liver grade increases with obesity, as indicated by anthropometric measures and adipocyte size. Compared to Caucasians, South Asians have a higher amount of total body 


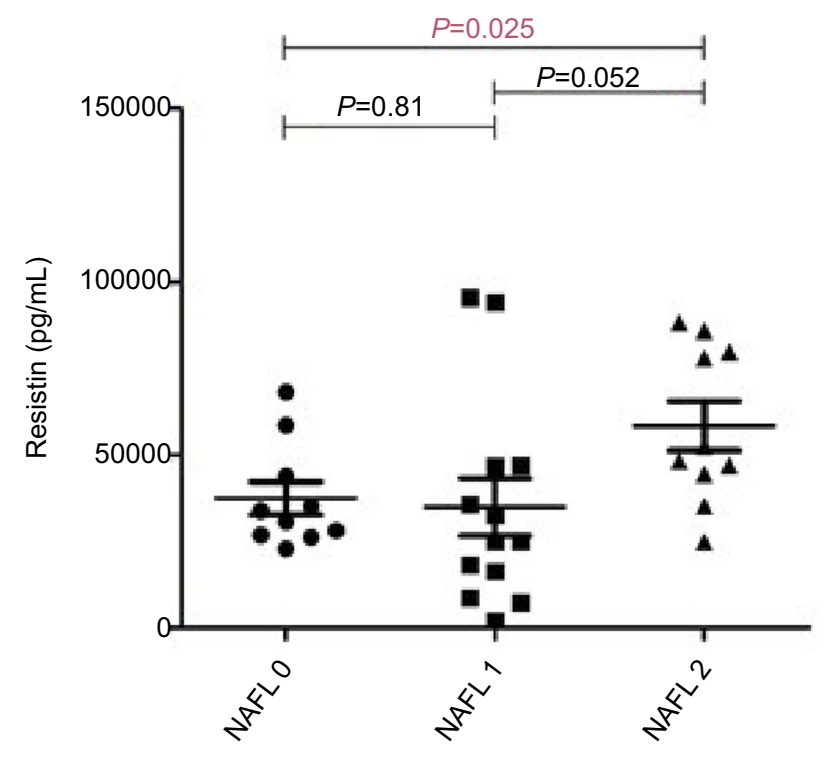

Figure 4 Elevated resistin associated with nonalcoholic fatty liver (NAFL) grade 2. Participants with fatty liver had serum resistin values that were trending significance. Participants with NAFL 2 had a significantly higher serum resistin as shown by post-hoc analyses, which also indicate increases in serum resistin between NAFL I and NAFL 2. No difference was found between NAFL 0 and NAFL I. Hepatic steatosis was assessed using ultrasound. $n=10-13$ per group. ANOVA with Bonferroni-Holm corrections analyses was performed.

fat for a given $\mathrm{BMI},{ }^{20}$ and thus lower values were established for BMI classifications (overweight, BMI $\geq 23 \mathrm{~kg} / \mathrm{m}^{2}$, obese BMI $\geq 27.49 \mathrm{~kg} / \mathrm{m}^{2}$ ) in this population. ${ }^{36}$ Furthermore, at a given total body fat, South Asians have higher total adipose tissue, including subcutaneous adipose tissue and visceral adipose tissue, compared to Caucasians. ${ }^{21}$

There is strong evidence that obesity-associated metabolic abnormalities occur at lower absolute amounts of total body fat in this population. ${ }^{37}$ It has been suggested that South Asians develop abdominal obesity earlier than Caucasians due to inefficient TG storage in the superficial subcutaneous adipose tissue compartment, which is the primary storage compartment. ${ }^{38}$ Increases in other compartments - the deep subcutaneous and visceral adipose tissue - as seen in South Asians, increases fatty acid flux and thus, the amount of fatty acids delivered to the liver. ${ }^{39}$ Indeed, liver steatosis is linked with increased adipocyte size and overall increased visceral adiposity. ${ }^{40}$

Earlier utilization of the secondary adipose tissue compartments explains greater values for anthropometric measures and justifies an increase in BMI, WC, HC, and both abdominal subcutaneous and visceral (omental) adipocyte size in our participants with NAFLD. The significant increase in subcutaneous abdominal adipocyte size with NAFLD is novel, especially the trending increase between NAFL 1 and NAFL $2(p=0.054)$ seen in our participants.
Interestingly, waist-to-hip ratio did not increase with increasing fatty liver. Invariably, previous studies have shown a weaker correlation with waist-to-hip ratio and metabolic abnormalities in this population. ${ }^{41}$

Surprisingly, lipoprotein profiles of our participants did not increase with the degree of fatty liver. This was unexpected since an atherogenic lipoprotein profile is often seen in South Asians with increased abdominal adiposity ${ }^{42}$ and has been linked with fatty liver in previous studies. ${ }^{43}$ Lipid values of our participants do compare to those reported in other studies, showing lower LDL in South Asians compared to Caucasians. ${ }^{44}$ Furthermore, low HDL and high TGs are common to dyslipidemia in South Asians. ${ }^{45,46}$ Consistent with previous studies, HDL averages for our participants were low $(<40 \mathrm{mg} / \mathrm{dL})$; however, interestingly, TG levels were not elevated (normal $\leq 150 \mathrm{mg} / \mathrm{dL}$ ) in our participants. Perhaps, the most interesting, when considering lipid panel results, is the lower TG levels and lack of correlation with a fatty liver degree in our participants. However, this has been reported in other studies ${ }^{47}$ and could be due to overall decreased VLDL hepatic output ${ }^{48}$ and increased hepatic TG storage.

With excessive nutrient intake, impaired adipogenesis leads to adipose tissue metabolic and immunologic dysfunction. ${ }^{12,49}$ Despite increasing adipocyte size with increasing NAFL grade, we did not see significant increases in the proinflammatory markers (IL-6, MCP-1, and hsCRP) evaluated in our study. CRP, which is strongly associated with central obesity in South Asians, ${ }^{50}$ was identified as a weak predictor of NAFLD. ${ }^{51}$ Accordingly, others have also reported no difference in hsCRP with NAFLD. ${ }^{52}$ Taking into account the insignificant findings regarding inflammation and AGT as well as enzymes for liver health, including ALT and AST, we suggest that individuals in our study must have stages of NAFLD that are not considered severe. ${ }^{53}$ This is supported in our findings for AST, which showed the largest increase in the NAFL 2 group. Furthermore, it may be that the threshold at which enlarged adipocytes contribute to systemic inflammation has not been reached. ${ }^{54}$

Additionally, insulin resistance is a common metabolic dysfunction associated with low-grade inflammation and impaired adipogenesis. ${ }^{12}$ However, serum insulin and HOMA calculations were not significantly increased with increasing NAFL grade in our study. Hyperglycemia, indicated by increased FBG, was seen in our participants with NAFLD. This is in contradiction to others who have reported no difference in fasting glucose but increased 
insulin and overall insulin resistance susceptibility in individuals with NAFLD. ${ }^{43,52}$ Others have shown BCAA alterations, ${ }^{34}$ decreased adiponectin, and increased leptin $^{55}$ to be associated with insulin resistance in South Asians; thus, it is not surprising that we did not see differences in our study.

Resistin is a hormone produced primarily by macrophages in humans and is positively correlated with obesity and insulin resistance ${ }^{56}$ due to its proinflammatory properties. ${ }^{57}$ The role of resistin in the pathogenesis of hepatic and systemic insulin resistance is well established in animals but is controversial in humans. ${ }^{58,59}$ Others have reported higher serum resistin levels in individuals with fatty liver and levels that parallel increasing fatty liver grade. ${ }^{52,60}$ Thus, resistin may participate in the pathways underlying liver damage and the progression of simple steatosis to steatohepatitis. ${ }^{52,61}$ Interestingly, in our study, resistin was trending significance, with the biggest increase in the NAFL 2 group. Since resistin stimulates hepatic glucose output, ${ }^{59,62,63}$ it may contribute to the elevated FBG seen in our study.

There are a few noteworthy limitations of the current study. The first limitation in our study is the small size of the study population. Despite this, several findings reached statistical significance when adjusting for the multiple comparisons performed. Sample size was calculated a priori, and we estimated 40 participants in each NAFL group would be needed in order to reach significance in those variables found nominally significant. Further, our convenience sample only included female participants, thus limiting the translation of our findings to males within this population. Third, liver biopsy is considered the gold standard for NAFLD diagnosis. Liver ultrasonography is a limitation in this study because it cannot differentiate between fibrosis and fatty changes. ${ }^{64}$ However, TG deposit is the most common pathological feature of NAFLD ${ }^{65}$ and most likely the dividing factor among our grades of NAFL. Interestingly, results from our study are similar if statistical analyses are conducted based on grouping (ie, the control [NAFL 0], normal liver echogenicity vs NAFL [both NAFL 1 and NAFL 2 group combined]) or alterations in liver echogenicity. Fourth, body composition analysis would have strengthened our discussion on adipocyte size and its relation to increasing NAFL grade. Additionally, utilizing the hyperinsulinemic-euglycemic clamp method or performing a glucose tolerance test on participants in our study would have aided in our understanding of dysglycemia in relation to increasing NAFL grade. Last, while dietary intake was collected, our interpretation and application to NAFL groups were limited. Diet could have significant implications in this population, given the shift to higher fat and fructose consumption in this population, as well as the known benefits of a Mediterranean diet and antioxidant consumption in NAFLD. ${ }^{17,66,67}$

\section{Conclusion}

It is well established that the associations of adiposity and health outcomes may differ between Asian and European populations. ${ }^{36}$ In the current study, we have shown that increases in adiposity are associated with NAFLD in South Asian women. Specifically, WC may be the best indicator of NAFLD progression in this population. Furthermore, increased subcutaneous as well as visceral fat deposition, elevated fasting glucose, and serum resistin could be important in the pathogenesis of NAFLD in South Asian women.

\section{Abbreviation list}

AGT, angiotensinogen; ALT, alanine aminotransferase; AST, aspartate aminotransferase; BMI, body mass index; FBG, fasting blood glucose; FFA, free fatty acids; HMW, high molecular weight; hsCRP, high-sensitivity $\mathrm{C}$-reactive protein; HC, hip circumference; MCP-1, monocyte chemoattractant protein 1; NAFL, nonalcoholic fatty liver; NAFLD, nonalcoholic fatty liver disease; NASH, nonalcoholic steatohepatitis; TG, triglyceride; WC, waist circumference.

\section{Acknowledgments}

We would like to acknowledge Mr. Shane Scoggin, Mr. K. P.G. Karunaratna, Mr. G.H.D.S. Chandraprabha, and Mr. P.U.B. Harangala for their technical assistance. This research was funded by an International Research/ Development Seed Grant from the Office of International Affairs at Texas Tech University, an International Research Seed Grant from the College of Human Sciences at Texas Tech University, and the Obesity Research Cluster at Texas Tech University. N.M.M. is in part supported by the National Institutes of Health National Center for Complementary and Integrative Health (NIH NCCIH) award number 1 R15 AT00887901A1. K.A.S. is a United States Department of Agriculture (USDA) National Institute of Food and Agriculture (NIFA) Fellow supported by an Agriculture and Food Research Initiative's (AFRI) Food, Agriculture, Natural Resources, and Human Sciences Education and Literacy Initiative (ELI) Predoctoral Fellowship under award 
number 2017-67011-26029. N.S.K. was funded by the International Research Center, University of Peradeniya, Sri Lanka (InRC/2015/01).

\section{Disclosure}

Dr. John A Dawson reports grants from American Egg Board, received travel reimbursements and conference fee from American Society for Nutrition outside the submitted work. The authors report no other conflicts of interest in this work.

\section{References}

1. Vernon G, Baranova A, Younossi ZM. Systematic review: the epidemiology and natural history of non-alcoholic fatty liver disease and non-alcoholic steatohepatitis in adults. Aliment Pharmacol Ther. 2011;34(3):274-285. doi:10.1111/j.1365-2036.2011.04724.x

2. Gupta R, Bhangoo A, Matthews NA, et al. The prevalence of non-alcoholic fatty liver disease and metabolic syndrome in obese children. J Pediatr Endocrinol Metab. 2011;24(11-12):907-911. doi:10.1515/JPEM.2011.282

3. Tominaga K, Fujimoto E, Suzuki K, Hayashi M, Ichikawa M, Inaba Y. Prevalence of non-alcoholic fatty liver disease in children and relationship to metabolic syndrome, insulin resistance, and waist circumference. Environ Health Prev Med. 2009;14(2):142-149. doi:10.1007/s12199-008-0074-5

4. Younossi Z, Tacke F, Arrese M, et al. Global perspectives on non-alcoholic fatty liver disease and non-alcoholic steatohepatitis. Hepatology. 2018. doi:10.1002/hep.30251

5. Angulo P. GI epidemiology: nonalcoholic fatty liver disease. Aliment Pharmacol Ther. 2007;25(8):883-889. doi:10.1111/j.13652036.2007.03246.x

6. Hagstrom H, Nasr P, Ekstedt M, et al. Fibrosis stage but not NASH predicts mortality and time to development of severe liver disease in biopsy-proven NAFLD. J Hepatol. 2017. doi:10.1016/j.jhep.2017.07.027

7. Milic S, Stimac D. Nonalcoholic fatty liver disease/steatohepatitis: epidemiology, pathogenesis, clinical presentation and treatment. Dig Dis. 2012;30(2):158-162. doi:10.1159/000336669

8. Youssef W, McCullough AJ. Diabetes mellitus, obesity, and hepatic steatosis. Semin Gastrointest Dis. 2002;13(1):17-30.

9. Barrows BR, Parks EJ. Contributions of different fatty acid sources to very low-density lipoprotein-triacylglycerol in the fasted and fed states. $J$ Clin Endocrinol Metab. 2006;91(4):1446-1452. doi:10.1210/jc.2005-1709

10. Kashyap S, Belfort R, Gastaldelli A, et al. A sustained increase in plasma free fatty acids impairs insulin secretion in nondiabetic subjects genetically predisposed to develop type 2 diabetes. Diabetes. 2003;52(10):2461-2474

11. Polyzos SA, Kountouras J, Mantzoros CS. Adipokines in nonalcoholic fatty liver disease. Metabolism. 2016;65(8):1062-1079. doi:10.1016/j.metabol.2015.11.006

12. Kalupahana NS, Claycombe KJ, Moustaid-Moussa N. (n-3) Fatty acids alleviate adipose tissue inflammation and insulin resistance: mechanistic insights. Adv Nutr. 2011;2(4):304-316. doi:10.3945/an.111.000505

13. Goldstein BJ. Insulin resistance as the core defect in type 2 diabetes mellitus. Am J Cardiol. 2002;90(5a):3g-10g.

14. Samuel VT, Shulman GI. Nonalcoholic Fatty liver disease as a nexus of metabolic and hepatic diseases. Cell Metab. 2018;27(1):22-41. doi:10.1016/j.cmet.2017.08.002

15. Browning JD, Szczepaniak LS, Dobbins R, et al. Prevalence of hepatic steatosis in an urban population in the United States: impact of ethnicity Hepatology. 2004;40(6):1387-1395. doi:10.1002/hep.20466

16. Kim D, Kim WR. Nonobese fatty liver disease. Clin Gastroenterol Hepatol. 2017;15(4):474-485. doi:10.1016/j.cgh.2016.08.028
17. Fan JG, Kim SU, Wong VW. New trends on obesity and NAFLD in Asia. J Hepatol. 2017;67(4):862-873. doi:10.1016/j.jhep.2017.06.003

18. Katulanda P, Jayawardena MA, Sheriff MH, Constantine GR, Matthews DR. Prevalence of overweight and obesity in Sri Lankan adults. Obes Rev. 2010;11(11):751-756. doi:10.1111/j.1467789X.2010.00746.x

19. Younossi ZM, Koenig AB, Abdelatif D, Fazel Y, Henry L, Wymer M. Global epidemiology of nonalcoholic fatty liver disease-Metaanalytic assessment of prevalence, incidence, and outcomes. Hepatology. 2016;64(1):73-84. doi:10.1002/hep.28431

20. Deurenberg-Yap M, Schmidt G, van Staveren WA, Deurenberg P. The paradox of low body mass index and high body fat percentage among Chinese, Malays and Indians in Singapore. Int $J$ Obes Relat Metab Disord. 2000;24(8):1011-1017.

21. Lear SA, Humphries KH, Kohli S, Chockalingam A, Frohlich JJ, Birmingham CL. Visceral adipose tissue accumulation differs according to ethnic background: results of the Multicultural Community Health Assessment Trial (M-CHAT). Am J Clin Nutr. 2007;86 (2):353-359. doi:10.1093/ajen/86.2.353

22. Ghosh A. Interrelationship of waist circumference and subcutaneous fat with metabolic and blood pressure measures among Asian Indian men. Anthropol Anz. 2007;65(1):75-85.

23. McKeigue PM, Pierpoint T, Ferrie JE, Marmot MG. Relationship of glucose intolerance and hyperinsulinaemia to body fat pattern in south Asians and Europeans. Diabetologia. 1992;35(8):785-791.

24. Misra A, Pandey RM, Sinha S, Guleria R, Sridhar V, Dudeja V. Receiver operating characteristics curve analysis of body fat \& body mass index in dyslipidaemic Asian Indians. Indian J Med Res. 2003;117:170-179.

25. Patel PJ, Banh X, Horsfall LU, et al. Underappreciation of non-alcoholic fatty liver disease by primary care clinicians: limited awareness of surrogate markers of fibrosis. Intern Med J. 2018;48(2):144-151.

26. Saadeh S, Younossi ZM, Remer EM, et al. The utility of radiological imaging in nonalcoholic fatty liver disease. Gastroenterology. 2002;123(3):745-750.

27. Aruni AW, Lee J, Osbourne D, et al. VimA-dependent modulation of acetyl coenzyme A levels and lipid A biosynthesis can alter virulence in Porphyromonas gingivalis. Infect Immun. 2012;80(2):550-564. doi:10.1128/IAI.06062-11

28. Hung AM, Sundell MB, Plotnikova NE, et al. A pilot study of active vitamin D administration and insulin resistance in African American patients undergoing chronic hemodialysis. J Ren Nutr. 2013;23 (3):185-193. doi:10.1053/j.jrn.2012.06.005

29. Kirchner H, Nylen C, Laber S, et al. Altered promoter methylation of PDK4, IL1 B, IL6, and TNF after Roux-en Y gastric bypass. Surg Obes Relat Dis. 2014;10(4):671-678. doi:10.1016/j.soard.2013.12.019

30. Mujumdar PP, Duerksen-Hughes PJ, Firek AF, Hessinger DA. Longterm, progressive, aerobic training increases adiponectin in middle-aged, overweight, untrained males and females. Scand J Clin Lab Invest. 2011;71(2):101-107. doi:10.3109/00365513.2011.554995

31. Rastogi A, Shasthry SM, Agarwal A, et al. Non-alcoholic fatty liver disease - histological scoring systems: a large cohort single-center, evaluation study. APMIS. 2017;125(11):962-973. doi:10.1111/apm.12742

32. Kwak JH, Jun DW, Lee SM, et al. Lifestyle predictors of obese and non-obese patients with nonalcoholic fatty liver disease: A cross-sectional study. Clin Nutr. 2018;37(5):1550-1557.

33. Association AD. Classification and Diagnosis of Diabetes. Diabetes Care. 2017;40(Supplement 1):S11-S24. doi:10.2337/dc17-S005

34. Tillin T, Hughes AD, Wang Q, et al. Diabetes risk and amino acid profiles: cross-sectional and prospective analyses of ethnicity, amino acids and diabetes in a South Asian and European cohort from the SABRE (Southall And Brent REvisited) Study. Diabetologia. 2015;58(5):968-979. doi:10.1007/s00125-015-3517-8

35. Wu Y, Ma KL, Zhang Y, et al. Lipid disorder and intrahepatic renin-angiotensin system activation synergistically contribute to non-alcoholic fatty liver disease. Liver Int. 2016;36(10):1525-1534. doi:10.1111/liv.13131 
36. WHO Expert Consultation. Appropriate body-mass index for Asian populations and its implications for policy and intervention strategies. Lancet. 2004;363(9403):157-163. doi:10.1016/S0140-6736(03)15268-3

37. Forouhi NG. Ethnicity and the metabolic syndrome. In: Byrne CD, Wild SH, editors. The Metabolic Syndrome. Chichester: Wiley; 2005:43-84.

38. Sniderman AD, Bhopal R, Prabhakaran D, Sarrafzadegan N, Tchernof A. Why might South Asians be so susceptible to central obesity and its atherogenic consequences? The adipose tissue overflow hypothesis. Int J Epidemiol. 2007;36(1):220-225. doi:10.1093/ije/dyl245

39. Sniderman AD, Cianflone K, Arner P, Summers LK, Frayn KN. The adipocyte, fatty acid trapping, and atherogenesis. Arterioscler Thromb Vasc Biol. 1998;18(2):147-151.

40. Koska J, Stefan N, Permana PA, et al. Increased fat accumulation in liver may link insulin resistance with subcutaneous abdominal adipocyte enlargement, visceral adiposity, and hypoadiponectinemia in obese individuals. Am J Clin Nutr. 2008;87(2):295-302. doi:10.1093/ajcn/87.2.295

41. Jayawardana R, Ranasinghe P, Sheriff MH, Matthews DR, Katulanda P. Waist to height ratio: a better anthropometric marker of diabetes and cardio-metabolic risks in South Asian adults. Diabetes Res Clin Pract. 2013;99(3):292-299. doi:10.1016/j.diabres.2012.12.013

42. Bilen O, Kamal A, Virani SS. Lipoprotein abnormalities in South Asians and its association with cardiovascular disease: current state and future directions. World J Cardiol. 2016;8(3):247-257. doi:10.4330/wjc.v8.i3.247

43. Kalhan SC, Guo L, Edmison J, et al. Plasma metabolomic profile in nonalcoholic fatty liver disease. Metabolism. 2011;60(3):404-413. doi:10.1016/j.metabol.2010.03.006

44. Karthikeyan G, Teo KK, Islam S, et al. Lipid profile, plasma apolipoproteins, and risk of a first myocardial infarction among Asians: an analysis from the INTERHEART Study. J Am Coll Cardiol. 2009;53 (3):244-253. doi:10.1016/j.jacc.2008.09.041

45. Gupta R, Gupta VP, Sarna M, et al. Prevalence of coronary heart disease and risk factors in an urban Indian population: Jaipur heart watch-2. Indian Heart J. 2002;54(1):59-66.

46. Gupta R, Guptha S, Sharma KK, Gupta A, Deedwania P. Regional variations in cardiovascular risk factors in India: india heart watch. World J Cardiol. 2012;4(4):112-120. doi:10.4330/wjc.v4.i4.112

47. Papandreou C, Bullo M, Tinahones FJ, et al. Serum metabolites in non-alcoholic fatty-liver disease development or reversion; a targeted metabolomic approach within the PREDIMED trial. Nutr Metab (Lond). 2017;14:58. doi:10.1186/s12986-017-0213-3

48. Lucero D, Miksztowicz V, Gualano G, et al. Nonalcoholic fatty liver disease associated with metabolic syndrome: influence of liver fibrosis stages on characteristics of very low-density lipoproteins. Clin Chim Acta. 2017;473:1-8. doi:10.1016/j.cca.2017.08.006

49. Skurk T, Alberti-Huber C, Herder C, Hauner H. Relationship between adipocyte size and adipokine expression and secretion. $J$ Clin Endocrinol Metab. 2007;92(3):1023-1033. doi:10.1210/jc.2006-1055

50. Forouhi NG, Sattar N, McKeigue PM. Relation of C-reactive protein to body fat distribution and features of the metabolic syndrome in Europeans and South Asians. Int $J$ Obes Relat Metab Disord. 2001;25(9):1327-1331. doi:10.1038/sj.ijo.0801723
51. Park SH, Kim BI, Yun JW, et al. Insulin resistance and C-reactive protein as independent risk factors for non-alcoholic fatty liver disease in non-obese Asian men. J Gastroenterol Hepatol. 2004;19 (6):694-698. doi:10.1111/j.1440-1746.2004.03362.x

52. Pagano C, Soardo G, Pilon C, et al. Increased serum resistin in nonalcoholic fatty liver disease is related to liver disease severity and not to insulin resistance. J Clin Endocrinol Metab. 2006;91 (3):1081-1086. doi:10.1210/jc.2005-1056

53. Wilfred de Alwis NM, Day CP. Genes and nonalcoholic fatty liver disease. Curr Diab Rep. 2008;8(2):156-163.

54. Kloting N, Bluher M. Adipocyte dysfunction, inflammation and metabolic syndrome. Rev Endocr Metab Disord. 2014;15 (4):277-287. doi:10.1007/s11154-014-9301-0

55. Wasim H, Al-Daghri NM, Chetty R, McTernan PG, Barnett AH, Kumar S. Relationship of serum adiponectin and resistin to glucose intolerance and fat topography in South-Asians. Cardiovasc Diabetol. 2006;5:10. doi:10.1186/1475-2840-5-10

56. Arner P. The adipocyte in insulin resistance: key molecules and the impact of the thiazolidinediones. Trends Endocrinol Metab. 2003;14 (3):137-145

57. Bokarewa M, Nagaev I, Dahlberg L, Smith U, Tarkowski A. Resistin, an adipokine with potent proinflammatory properties. J Immunol. 2005;174(9):5789-5795.

58. Steppan CM, Bailey ST, Bhat S, et al. The hormone resistin links obesity to diabetes. Nature. 2001;409(6818):307-312. doi:10.1038/ 35053000

59. Muse ED, Obici S, Bhanot S, et al. Role of resistin in diet-induced hepatic insulin resistance. J Clin Invest. 2004;114(2):232-239. doi:10.1172/JCI21270

60. Murad A. HH, H Husein \& A Ayad. Serum resistin levels in nonalcoholic fatty liver disease and their relationship to severity of liver disease. JEMDSA. 2010;15(1):53-55. doi:10.1080/22201009.2010.10872225

61. Chitturi S, Farrell GC. Etiopathogenesis of nonalcoholic steatohepatitis. Semin Liver Dis. 2001;21(1):27-41.

62. Rangwala SM, Rich AS, Rhoades B, et al. Abnormal glucose homeostasis due to chronic hyperresistinemia. Diabetes. 2004;53 (8):1937-1941. 53.08.04.db04-0422

63. Banerjee RR, Rangwala SM, Shapiro JS, et al. Regulation of fasted blood glucose by resistin. Science. 2004;303(5661):1195-1198. doi:10.1126/science.1092341

64. Abd El-Kader SM, El-Den Ashmawy EM. Non-alcoholic fatty liver disease: the diagnosis and management. World J Hepatol. 2015;7 (6):846-858. doi:10.4254/wjh.v7.i6.846

65. Ludwig J, Viggiano TR, McGill DB, Oh BJ. Nonalcoholic steatohepatitis: mayo clinic experiences with a hitherto unnamed disease. Mayo Clin Proc. 1980;55(7):434-438.

66. Abenavoli L, Greco M, Milic N, et al. Effect of mediterranean diet and antioxidant formulation in non-alcoholic fatty liver disease: a randomized study. Nutrients. 2017;9:8. doi:10.3390/nu9080870

67. Suarez M, Boque N, Del Bas JM, Mayneris-Perxachs J, Arola L, Caimari A. Mediterranean diet and multi-ingredient-based interventions for the management of non-alcoholic fatty liver disease. Nutrients. 2017;9:10. doi:10.3390/nu9101052

Diabetes, Metabolic Syndrome and Obesity: Targets and Therapy

\section{Dovepress}

\section{Publish your work in this journal}

Diabetes, Metabolic Syndrome and Obesity: Targets and Therapy is an international, peer-reviewed open-access journal committed to the rapid publication of the latest laboratory and clinical findings in the fields of diabetes, metabolic syndrome and obesity research. Original research, review, case reports, hypothesis formation, expert opinion and commentaries are all considered for publication. The manuscript management system is completely online and includes a very quick and fair peer-review system, which is all easy to use. Visit http://www.dovepress.com/testimonials.php to read real quotes from published authors. 